\title{
Simplicity-Expressiveness Tradeoffs in Mechanism Design
}

\section{Citation}

Dütting, Paul, Felix Fischer and David C. Parkes. Forthcoming. Simplicity-Expressiveness Tradeoffs in Mechanism Design. In Proceedings of the 12th ACM Conference on Electronic Commerce, June 5-9, 2011, San Jose, CA.

\section{Permanent link}

http://nrs.harvard.edu/urn-3:HUL.InstRepos:4892934

\section{Terms of Use}

This article was downloaded from Harvard University's DASH repository, and is made available under the terms and conditions applicable to Open Access Policy Articles, as set forth at http:// nrs.harvard.edu/urn-3:HUL.InstRepos:dash.current.terms-of-use\#OAP

\section{Share Your Story}

The Harvard community has made this article openly available.

Please share how this access benefits you. Submit a story.

Accessibility 


\section{Simplicity-Expressiveness Tradeoffs in Mechanism Design}

\author{
Paul Dütting \\ Ecole Polytechnique Fédérale \\ de Lausanne (EPFL) \\ $\mathrm{CH}-1015$ Lausanne, Switzerland \\ paul.duetting@epfl.ch
}

\author{
Felix Fischer $^{\dagger}$ \\ School of Engineering and \\ Applied Sciences \\ Harvard University \\ Cambridge, MA 02138, USA \\ fischerf@seas.harvard.edu
}

\author{
David C. Parkes \\ School of Engineering and \\ Applied Sciences \\ Harvard University \\ Cambridge, MA 02138, USA \\ parkes@eecs.harvard.edu
}

Everything should be made as simple as possible, but no simpler.

- Albert Einstein

\begin{abstract}
A fundamental result in mechanism design theory, the socalled revelation principle, asserts that for many questions concerning the existence of mechanisms with a given outcome one can restrict attention to truthful direct-revelation mechanisms. In practice, however, many mechanisms use a restricted message space. This motivates the study of the tradeoffs involved in choosing simplified mechanisms, which can sometimes bring benefits in precluding bad or promoting good equilibria, and other times impose costs on welfare and revenue. We study the simplicity-expressiveness tradeoff in two representative settings, sponsored search auctions and combinatorial auctions, each being a canonical example for complete information and incomplete information analysis, respectively. We observe that the amount of information available to the agents plays an important role for the tradeoff between simplicity and expressiveness.
\end{abstract}

\section{Categories and Subject Descriptors}

F.2 [Theory of Computation]: Analysis of Algorithms and Problem Complexity; J.4 [Computer Applications]: Social and Behavioral Sciences-Economics

\section{General Terms}

Algorithms, Economics, Theory

\section{Keywords}

Simplified Mechanisms, Expressiveness

\footnotetext{
* Research supported by a EURYI award.

${ }^{\dagger}$ Research supported by DFG grant FI 1664/1-1.
}

Permission to make digital or hard copies of all or part of this work for personal or classroom use is granted without fee provided that copies are not made or distributed for profit or commercial advantage and that copies bear this notice and the full citation on the first page. To copy otherwise, to republish, to post on servers or to redistribute to lists, requires prior specific permission and/or a fee.

EC'11, June 5-9, 2011, San Jose, California, USA.

Copyright 2011 ACM 978-1-4503-0261-6/11/06 ...\$10.00.

\section{INTRODUCTION}

The revelation principle states that any equilibrium outcome of any mechanism can be obtained in a truthful equilibrium of a direct-revelation mechanism, i.e., an equilibrium in which agents truthfully reveal their types. In practice, however, the assumptions underlying the revelation principle often fail: other equilibria may exist besides the truthful one, or computational limitations may interfere. As a consequence, many practical mechanisms use a restricted message space. This motivates the study of the tradeoffs involved in choosing among mechanisms with different degrees of expressiveness. Despite their practical importance, these tradeoffs are currently only poorly understood.

In sponsored search auctions, and adopting a complete information analysis, allowing every agent $i$ to submit a valuation $v_{i j}$ for each slot $j$ means that both the Vickrey-ClarkeGroves (VCG) mechanism and the Generalized Second Price (GSP) mechanism always admit a Nash equilibrium with zero revenue [22]. If agents face a small cost for submitting a non-zero bid, this becomes the unique equilibrium. If instead agent $i$ is asked for a single bid $b_{i}$, and his bid for slot $j$ is derived by multiplying $b_{i}$ with a slot-specific click-through rate $\alpha_{j}$, the zero-revenue equilibria are eliminated. More surprisingly, this simplification does not introduce new equilibria (even when $\alpha_{j}$ is not correct for every agent $i$ ), so minimum revenue over all Nash equilibria is strictly greater for the simplification than for the original mechanism. Moreover, if valuations can actually be decomposed into an agent-specific valuation $v_{i}$ and click-through rates $\alpha_{j}$, the simplification still has an efficient Nash equilibrium. Milgrom [22] concluded that simplification can be beneficial and need not come at a cost.

For combinatorial auctions, and adopting an incomplete information analysis, the maximum social welfare over all outcomes of a mechanism strictly increases with expressiveness, for a particular measure of expressiveness based on notions from computational learning theory [4]. Implicit in the result of Benisch et al. [4] is the conclusion that more expressiveness is generally desirable, as it allows a mechanism to achieve a more efficient outcome in more instances of the problem.

Each of these results tells only part of the story. While Milgrom's results on the benefits of simplicity are developed within an equilibrium framework and can be extended beyond sponsored search auctions, they critically require settings with complete information amongst agents in order to preclude bad equilibria while retaining good ones. In particular, Milgrom does not consider the potential loss in 
efficiency or revenue that can occur when agents are ignorant of each other's valuations when deciding how to bid within a simplified bidding language. Benisch et al., on the other hand, develop their results on the benefits of expressiveness (and thus the cost of simplicity) in an incomplete information context, but largely in the absence of equilibrium considerations. ${ }^{1}$ In particular, these authors do not consider the potential problems that can occur due to the existence of bad equilibria in expressive mechanisms.

Our Contribution The contribution of this paper is twofold. On a conceptual level, we analyze how different properties of a simplification affect the set of equilibria of a mechanism in both complete and incomplete information settings and argue that well-chosen simplifications can have a positive impact on the set of equilibria as a whole; either by precluding undesirable equilibria or by promoting desirable equilibria. We thus extend Milgrom's emphasis on simplification as a tool that enables equilibrium selection. On a technical level, we analyze simplified mechanisms for sponsored search auctions with complete information and combinatorial auctions with incomplete information.

An important property when analyzing the impact of simplification on the set of equilibria is tightness [22], which requires that no additional equilibria are introduced. We observe that tightness can be achieved equally well in complete and incomplete information settings and give a sufficient condition. ${ }^{2}$ Complementary to tightness is a property we call totality, which requires all equilibria of the original mechanism to be preserved. To the end of equilibrium selection, totality needs to be relaxed. Particular relaxations we consider in this paper are the preservation of the VCG outcome, i.e., the outcome obtained in the dominant strategy equilibrium of the fully expressive VCG mechanism, and the existence of an equilibrium with a certain amount of social welfare or revenue relative to the VCG outcome. In addition, one might require that the latter property holds for every equilibrium of the simplified mechanism.

In the context of sponsored search, one reason to prefer a simplification is to preclude the zero-revenue equilibria discussed above. Another interesting property of a simplified GSP mechanism is that it preserves the VCG outcome even when the assumed click-through rates $\alpha_{j}$ are inexact. Recognizing that this claim cannot be made for the VCG mechanism under the same simplification, Milgrom [22] uses this as an argument for the superiority of the GSP mechanism. But, this result that GSP is Vickrey-preserving requires an unnatural condition on the relationship between the assumed click-through rates and prices and thus agents' bids, and moreover does not preclude alternate simplifications of VCG that succeed in being Vickrey-preserving. In addition, we observe that the simplifications can still suffer arbitrarily low revenue in some equilibrium, in comparison with the VCG revenue.

In our analysis of sponsored search, we identify a simplified GSP mechanism that preserves the VCG outcome without requiring any knowledge of the actual click-through rates, precludes zero-revenue equilibria, and always recov-

\footnotetext{
${ }^{1}$ The equilibrium analysis that Benisch et al. provide is in regard to identifying a particular mechanism design in which the maximum social welfare achievable in any outcome can be achieved in a particular Bayes-Nash equilibrium.

${ }^{2}$ This condition was already considered by Milgrom, but only in the complete information case.
}

ers at least half of the VCG payments for all slots but the first. For simplified VCG mechanisms, we obtain a strong negative result: every simplification of VCG that supports the (efficient) VCG outcome in some equilibrium also has an equilibrium in which revenue is arbitrarily smaller than in the VCG outcome.

In the context of combinatorial auctions, paradigmatic of course of settings with incomplete information, we first recall the previous observations by Holzman et al. [15, 16] in regard to the existence of multiple, non-truthful, ex-post equilibria of the VCG mechanism, each of which offers different welfare and revenue properties. In particular, if one assumes that participants will select equilibria with a particular (maximum) number of bids, social welfare can differ greatly among the different equilibria, revenue can be zero for some of them, and the existence of multiple Pareto optimal equilibria can make equilibrium selection hard to impossible for participants.

Focusing again on tight simplifications, we connect the analysis of Holzman et al. [15, 16] with tightness, by establishing that a simplification is tight if and only if bids are restricted to a subset $\Sigma$ of the bundles with a quasi-field structure $[15,16]$, with values for the other bundles derived as the maximum value of any contained bundle. Through insisting on a tight simplification, we ensure that the worst-case behavior is no worse than that of the fully expressive VCG mechanism, even when $\Sigma$ (although simplified) contains too many bundles for agents to bid on all of them. Moreover, using a quasi-field simplification ensures that agents do not experience regret with respect to the bidding language, in the sense of wanting to send a message ex post that was precluded. Finally, as any restriction of the bids to a subset of the bundles, restricting the bids to a quasi-field $\Sigma$ yields a mechanism that is maximal in range [23] and makes it a dominant strategy equilibrium for the agents to bid truthfully on these bundles. Simplification thus enables the mechanism designer to guide equilibrium selection, and our results suggest that the presence or absence of such guidance can have a significant impact on the economic properties of the mechanism.

The informational assumptions underlying our analysis are crucial, and the amount of information available to the agents plays an important role for the tradeoff between simplicity and expressiveness. In the sponsored search setting, both the existence of a zero-revenue equilibrium in the expressive mechanism, and the existence of the desirable equilibrium in the simplification, rely on the assumption of complete information. In other words, agents can on one hand use information about each others' types to coordinate and harm the auctioneer, but on the other hand, the same information guarantees that simplified mechanisms retain the desirable equilibria of the expressive mechanism. In combinatorial auctions the contrast is equally stark: while bids on every single package may be required to sustain an efficient equilibrium in the incomplete information setting, we show that such an equilibrium can be obtained with a number of bids that is quadratic in the number of agents, and potentially exponentially smaller than the number of bundles, given that agents have complete information.

Related Work Several authors have criticized the revelation principle because it does not take computational aspects of mechanisms into account. In this context, Conitzer and Sandholm [10] consider sequential mechanisms that reduce 
the amount of communication, and non-truthful mechanisms that shift the computational burden of executing the mechanism, and the potential loss when it is executed suboptimally, from the designer to the agents. Hyafil and Boutilier $[17,18]$ propose to circumvent computational problems associated with direct type revelation via the automated design of partial-revelation mechanisms, and in particular study approximately incentive compatible mechanisms that do not make any assumptions about agents' preferences. This approach is very general, but also hard to analyze theoretically, with complex, regret-based algorithms.

Blumrosen et al. [6] and Feldman and Blumrosen [12] consider settings with one-dimensional types and ask how much welfare and revenue can be achieved by mechanisms with a bounded message space. By contrast, we study mechanisms with message spaces that grow in some parameter of the problem and may even have infinite size, and obtain results both for one-dimensional and multi-dimensional types.

A different notion of simplicity of a mechanism was considered by Babaioff and Roughgarden [3]: the authors show that among all payment rules that guarantee an efficient equilibrium when ranking agents according to their bids, the GSP payment rule is optimally simple in the sense that prices depend on bids in a minimal way.

Shakkottai et al. [25] study the tradeoff between simplicity and revenue in the context of pricing rules for communication networks and define the "price of simplicity" as the ratio between the revenue of a very simple pricing rule and the maximum revenue that can be obtained.

\section{PRELIMINARIES}

A mechanism design problem is given by a set $N=$ $\{1,2, \ldots, n\}$ of agents that interact to select an element from a set $\Omega$ of outcomes. Agent $i \in N$ is associated with a type $\theta_{i}$ from a set $\Theta_{i}$ of possible types, representing private information held by this agent. We write $\theta=\left(\theta_{1}, \ldots, \theta_{n}\right)$ for a profile of types for the different agents, $\Theta=\prod_{i \in N} \Theta_{i}$ for the set of possible type profiles, and $\theta_{-i} \in \Theta_{-i}$ for a profile of types for all agents but $i$. Each agent $i \in N$ further employs preferences over $\Omega$, represented by a valuation function $v_{i}: \Omega \times \Theta_{i} \rightarrow \mathbb{R}$. The quality of an outcome $o \in \Omega$ is typically measured in terms of its social welfare, which is defined as the sum $\sum_{i \in N} v_{i}\left(o, \theta_{i}\right)$ of agents' valuations. An outcome that maximizes social welfare is also called efficient.

A mechanism is given by a tuple $(N, X, f, p)$, where $X=\prod_{i \in N} X_{i}$ is a set of message profiles, $f: X \rightarrow \Omega$ is an outcome function, and $p: X \rightarrow \mathbb{R}^{n}$ is a payment function. In this paper, we mostly restrict our attention to direct mechanisms, i.e., mechanisms where $X_{i} \subseteq \Theta_{i}$ for every $i \in N$. A direct mechanism $(N, X, f, p)$ with $X=\Theta$ is called efficient if for every $\theta \in \Theta, f(\theta)$ is an efficient outcome. Just as for type profiles, we write $x_{-i} \in X_{-i}$ for a profile of messages by all agents but $i$. We assume quasilinear preferences, i.e., the utility of agent $i$ given a message profile $x \in X$ is $u_{i}\left(x, \theta_{i}\right)=v_{i}\left(f(x), \theta_{i}\right)-p_{i}(x)$. The revenue achieved by mechanism $(N, X, f, p)$ for a message profile $x \in X$ is $\sum_{i \in N} p_{i}(x)$.

Game-theoretic reasoning is used to analyze how agents interact with a mechanism, a desirable criterion being stability according to some game-theoretic solution concept. We consider two different settings. In the complete information setting, agents are assumed to know the type of every other agent. A strategy of agent $i$ in this setting is a function $s_{i}: \Theta \rightarrow X_{i}$. In the (strict) incomplete information setting, agents have no information, not even distributional, about the types of the other agents. A strategy of agent $i$ in this setting thus becomes a function $s_{i}: \Theta_{i} \rightarrow X_{i}$.

The two most common solution concepts in the complete information setting are dominant strategy equilibrium and Nash equilibrium. A strategy $s_{i}: \Theta \rightarrow X_{i}$ is a dominant strategy if for every $\theta \in \Theta$, every $x_{-i} \in X_{-i}$, and every $x_{i} \in X_{i}$,

$$
u_{i}\left(\left(s_{i}(\theta), x_{-i}\right), \theta_{i}\right) \geq u_{i}\left(\left(x_{i}, x_{-i}\right), \theta_{i}\right) .
$$

A profile $s \in \prod_{i \in N} s_{i}$ of strategies $s_{i}: \Theta \rightarrow X_{i}$ is a Nash equilibrium if for every $\theta \in \Theta$, every $i \in N$, and every $s_{i}^{\prime}: \Theta \rightarrow X_{i}$,

$$
u_{i}\left(\left(s_{i}(\theta), s_{-i}(\theta)\right), \theta_{i}\right) \geq u_{i}\left(\left(s_{i}^{\prime}(\theta), s_{-i}(\theta)\right), \theta_{i}\right) .
$$

The existence of a dominant strategy $s_{i}: \Theta \rightarrow X_{i}$ always implies the existence of a dominant strategy $s_{i}^{\prime}: \Theta_{i} \rightarrow X_{i}$ that does not depend on the types of other agents. The solution concept of dominant strategy equilibrium thus carries over directly to the incomplete information setting. Formally, a strategy $s_{i}: \Theta_{i} \rightarrow X_{i}$ is a dominant strategy in the incomplete information setting if for every $\theta_{i} \in \Theta_{i}$, every $x_{-i} \in X_{-i}$, and every $x_{i} \in X_{i}$,

$$
u_{i}\left(\left(s_{i}\left(\theta_{i}\right), x_{-i}\right), \theta_{i}\right) \geq u_{i}\left(\left(x_{i}, x_{-i}\right), \theta_{i}\right) .
$$

The appropriate variant of the Nash equilibrium concept in that setting is that of an ex-post equilibrium. A profile $s \in$ $\prod_{i \in N} s_{i}$ of strategies $s_{i}: \Theta_{i} \rightarrow X_{i}$ is an ex-post equilibrium if for every $\theta \in \Theta$, every $i \in N$, and every $s_{i}^{\prime}: \Theta_{i} \rightarrow X_{i}$,

$$
u_{i}\left(\left(s_{i}\left(\theta_{i}\right), s_{-i}\left(\theta_{-i}\right)\right), \theta_{i}\right) \geq u_{i}\left(\left(s_{i}^{\prime}\left(\theta_{i}\right), s_{-i}\left(\theta_{-i}\right)\right), \theta_{i}\right) .
$$

We conclude this section with a direct mechanism due to Vickrey [27], Clarke [9], and Groves [14]. This mechanism starts from an efficient outcome function $f$ and computes each agent's payment according to the total value of the other agents, thus aligning his interests with that of society. Formally, mechanism $(N, X, f, p)$ is called Vickrey-ClarkeGroves (VCG) mechanism ${ }^{3}$ if $X=\Theta, f$ is efficient, and

$$
p_{i}(\theta)=\max _{o \in \Omega} \sum_{j \neq i} v_{j}\left(o, \theta_{j}\right)-\sum_{j \neq i} v_{j}\left(f(\theta), \theta_{j}\right) .
$$

In the VCG mechanism, revealing types $\theta \in \Theta$ truthfully is a dominant strategy equilibrium [14]. We will refer to the resulting outcome as the VCG outcome for $\theta$, and write $R^{V C G}(\theta)$ for the revenue obtained in this outcome.

\section{SIMPLIFICATIONS}

Our main object of study in this paper are simplifications of a mechanism obtained by restricting its message space. Consider a mechanism $M=(N, X, f, p)$. A mechanism $\hat{M}=$ $(N, \hat{X}, \hat{f}, \hat{p})$ will be called a simplification of $M$ if $\hat{X} \subseteq X$, $\left.\hat{f}\right|_{\hat{X}}=\left.f\right|_{\hat{X}}$, and $\left.\hat{p}\right|_{\hat{X}}=\left.p\right|_{\hat{X}}$.

We will naturally be interested in the set of outcomes that can be obtained in equilibrium, both in the original mechanism $M$ and the simplified mechanism $\hat{M}^{4}$ Milgrom [22]

\footnotetext{
${ }^{3}$ Actually, we consider a specific member of a whole family of VCG mechanisms, namely the one that uses the Clarke pivot rule.

${ }^{4}$ In the following, we will simply talk about equilibria without making a distinction between the different equilibrium
} 
defines a property he calls tightness, which requires that the simplification does not introduce any additional equilibria. More formally, simplification $\hat{M}$ will be called tight if every equilibrium of $\hat{M}$ is an equilibrium of $M .^{5}$ Tightness ensures that the simplified mechanism is at least as good as the original one with respect to the worst outcome obtained in any equilibrium. It does not by itself protect good equilibrium outcomes, and we will in fact see examples of tight simplifications that eliminate all ex-post equilibria.

A property that will be useful in the following is a variant of Milgrom's outcome closure for exact equilibria. It requires that for every agent, and for every choice of messages from the restricted message spaces of the other agents, it is optimal for the agent to choose a message from his restricted message space. More formally, a simplification $(N, \hat{X}, \hat{f}, \hat{p})$ of a mechanism $(N, X, f, p)$ satisfies outcome closure if for every $\theta \in \Theta$, every $i \in N$, every $\hat{x}_{-i} \in \hat{X}_{-i}$, and every $x_{i} \in X_{i}$ there exists $\hat{x}_{i} \in \hat{X}_{i}$ such that $u_{i}\left(\left(\hat{x}_{i}, \hat{x}_{-i}\right), \theta_{i}\right) \geq$ $u_{i}\left(\left(x_{i}, \hat{x}_{-i}\right), \theta_{i}\right)$.

This turns out to be sufficient for tightness in both the complete and incomplete information case. ${ }^{6}$

Proposition 1 (Milgrom [22]). Every simplification that satisfies outcome closure is tight with respect to both Nash and ex-post equilibria.

One way to guarantee good behavior in the best case is by requiring that a simplification $\hat{M}$ preserves all equilibria of the original mechanism $M$, in the sense that for every Nash equilibrium of $M$, there exists an equilibrium of $\hat{M}$ that yields the same outcome and payments. We will call a simplification satisfying this property total, and will return to total mechanisms in Section 6. To the end of equilibrium selection totality clearly needs to be relaxed, by requiring that only certain desirable outcomes are preserved. A typical desirable outcome in many settings is the VCG outcome. For example, although this outcome has some shortcomings in fully general combinatorial auction domains [2], it remains of significant interest in settings with unit-demand preferences, such as sponsored search. We will call simplification $\hat{M}$ Vickrey-preserving if for every $\theta \in \Theta$, it has an equilibrium that yields the VCG outcome for $\theta$.

\section{SPONSORED SEARCH AUCTIONS}

In sponsored search (see, e.g., [20]), the agents compete for elements of a set $S=\{1, \ldots, k\}$ of slots, where $k \leq n$. Each outcome corresponds to a one-to-one assignment of agents to slots, i.e., $\Omega \subseteq\{1, \ldots, n\}^{N}$ such that $o_{i} \neq o_{j}$ for all $o \in \Omega$ and $i, j \in\{1, \ldots, n\}$ with $i \neq j$. We will assume that $v\left(o, \theta_{i}\right)=0$ if $o_{i}>k$, and that there are no externalities, i.e., $v_{i}\left(o, \theta_{i}\right)=v_{i}\left(o^{\prime}, \theta_{i}\right)$ if $o_{i}=o_{i}^{\prime}$. In slight abuse of notation, we will write $v_{i}\left(j, \theta_{i}\right)$ for the valuation of agent $i$ for slot $j$.

We consider simplifications of two mechanisms, the Vickrey-Clarke-Groves (VCG) mechanism and the Generalized Second Price (GSP) mechanism, and analyze their

notions. Unless explicitly noted otherwise, our results concern Nash equilibria in the complete information case and ex-post equilibria in the incomplete information case.

${ }^{5}$ Milgrom [22] considers a slightly stronger notion of tightness defined with respect to (pure-strategy) $\epsilon$-Nash equilibria.

${ }^{6}$ Milgrom stated the result only for complete information, but the proof goes through for incomplete information as well. behavior for different spaces of type profiles, which we denote by $\Theta, \Theta^{>}$, and $\Theta^{\alpha}$. In $\Theta$, valuations can be arbitrary non-negative numbers. $\Theta^{>}$adds the restriction that valuations are strictly decreasing, i.e., $v_{i}\left(j, \theta_{i}\right)>v_{i}\left(j+1, \theta_{i}\right)$ for every $\theta \in \Theta^{>}, i \in N$, and $j \in\{1, \ldots, k-1\}$. Valuations in $\Theta^{\alpha}$ are assumed to arise from "clicks" associated with each slot and a valuation per click. In other words, there exists a fixed click-through rate vector $\alpha \in \mathbb{R}_{>}^{n}=\left\{\alpha^{\prime} \in \mathbb{R}^{n}\right.$ : $\alpha_{i}^{\prime}>\alpha_{j}^{\prime}$ if $\left.i<j\right\}$, which may or may not be known to the mechanism, and $v_{i}\left(j, \theta_{i}\right)=\alpha_{j} \cdot v_{i}\left(\theta_{i}\right)$ for some $v_{i}\left(\theta_{i}\right) \in \mathbb{R}_{\geq 0}$. Thus, $\alpha_{j}=0$ if $j>k$, and it will be convenient to assume that $\alpha_{1}=1$. We finally define $\Psi=\bigcup_{\alpha \in \mathbb{R}_{>}^{n}} \Theta^{\alpha}$, and observe that $\Psi \subset \Theta^{>} \subset \Theta$.

The message an agent $i \in N$ submits to the mechanisms thus corresponds to a vector of bids $x_{i, j} \in \mathbb{R}$ for slots $j \in S$. Given a message profile $x \in X$, the VCG mechanism assigns each agent $i$ a slot $f_{i}(x)=o_{i}$ so as to maximize $\sum_{i} x_{i, o_{i}}$, and charges that agent $p_{i}(x)=\max _{o^{\prime} \in \Omega} \sum_{j \neq i} x_{j, o_{j}^{\prime}}-\sum_{j \neq i} x_{j, o_{j}}$. The GSP mechanism is defined via a sequence of secondprice auctions for slots 1 through $k$ : slot $j$ is assigned to an agent $i$ with a maximum bid for that slot at a price equal to the second highest bid, both with respect to the set of agents who have not yet been assigned a slot, i.e., $f_{i}(x)=o_{i}$ such that $x_{i, j}=\max _{i^{\prime} \in N: o_{i^{\prime}} \geq j} x_{i^{\prime}, j}$ and $p_{i}(x)=$ $\max _{i^{\prime} \in N: o_{i^{\prime}}>j} x_{i, j}$.

\subsection{Envy-Freeness and Efficiency}

The original analysis of GSP due to Edelman et al. [11] and Varian [26] focuses on equilibria that are "locally envyfree." ${ }^{\prime 7}$ Assume that $\theta \in \Theta^{\alpha}$, and consider an outcome in which agent $i$ is assigned slot $i$, for all $i \in N$. Such an outcome is called locally envy-free if, in addition to being a Nash equilibrium, no agent could increase his utility by exchanging bids with the agent assigned the slot directly above him, i.e., if for every $i \in\{2, \ldots, n\}, \alpha_{i} \cdot v_{i}\left(\theta_{i}\right)-p_{i} \geq$ $\alpha_{i-1} \cdot v_{i}\left(\theta_{i}\right)-p_{i-1}$. Restricting attention to envy-free equilibria immediately solves all revenue problems: as Edelman et al. point out, revenue in any locally envy-free equilibrium is at least as high as that in the dominant-strategy equilibrium of the VCG auction. Conversely, Milgrom's observation concerning zero-revenue equilibria contains an implicit critique of the assumptions underlying the restriction to equilibria that are envy-free. It will be instructive to make this critique explicit.

Edelman et al. argue that an equilibrium where some agent $i$ envies some other agent $j$ assigned the next higher slot is not a reasonable rest point of the bidding process, because agent $i$ might increase the price paid by agent $j$ without the danger of harming his own utility should $j$ retaliate. There are two problems with this line of reasoning. First, it is not clear why agent $j$ should retaliate, especially if he is worse off by doing so. Second, agent $i$ might in fact have a very good reason not to increase the price paid by $j$, like a desire to keep prices low in the long run through tacit collusion.

We may instead ask under what conditions there exists a bid $x_{i, j}$ for agent $i$ on slot $j$ such that (i) agent $j$ is forced out of slot $j$, in the sense that it becomes a better response for $j$ to underbid $i$, and (ii) agent $i$ is strictly better off after this response by $j$ than at present. This is the case exactly

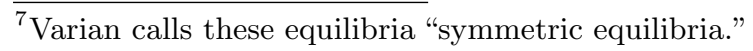


when

$$
\begin{aligned}
& \alpha_{i} \cdot v_{j}\left(\theta_{j}\right)-p_{i}>\alpha_{j} \cdot v_{j}\left(\theta_{j}\right)-x_{i, j} \quad \text { and } \\
& \alpha_{j} \cdot v_{i}\left(\theta_{i}\right)-x_{i, j}>\alpha_{i} \cdot v_{i}\left(\theta_{i}\right)-p_{i},
\end{aligned}
$$

where $p_{i}$ is the price currently paid by agent $i$. The second inequality assumes that $j$ will respond by bidding just below the bid $x_{i, j}$ of agent $i$, such that this becomes $i$ 's new price.

An equilibrium that does not allows deviations as above will be called two-step stable. It turns out that two-step stability exactly characterizes the set of efficient equilibria.

Proposition 2. Let $\alpha \in \mathbb{R}_{>}^{n}, \theta \in \Theta^{\alpha}$. Then, an equilibrium is two-step stable if and only it is efficient.

Proof. Rewriting (1), it must hold that

$$
\begin{aligned}
& x_{i, j}>\left(\alpha_{j}-\alpha_{i}\right) \cdot v_{j}\left(\theta_{j}\right)+p_{i} \quad \text { and } \\
& x_{i, j}<\left(\alpha_{j}-\alpha_{i}\right) \cdot v_{i}\left(\theta_{i}\right)+p_{i} .
\end{aligned}
$$

Clearly, a bid $x_{i, j}$ with this property exists if and only if $v_{i}\left(\theta_{i}\right)>v_{j}\left(\theta_{j}\right)$. In turn, agents $i$ and $j$ such that $v_{i}\left(\theta_{i}\right)>$ $v_{j}\left(\theta_{j}\right)$ exist if and only if the current assignment is inefficient.

This result provides a very strong argument against inefficient equilibria as rest points of the bidding process, much stronger than the argument against equilibria that are not envy-free. In the context of sponsored search auctions we will therefore restrict our attention to efficient equilibria. It is worth noting at this point that the set of efficient equilibria forms a strict superset of the set of locally envy-free equilibria, and in particular contains the zero-revenue equilibria identified by Milgrom and discussed next.

\subsection{Comments on Milgrom's Analysis}

Milgrom [22] observed that for every profile of agent types, both VCG and GSP have a Nash equilibrium that yields zero revenue, and that this equilibrium in fact becomes the unique equilibrium if there is a small cost associated with submitting a positive bid. To alleviate this fact, he proposed to restrict the message space of both VCG and GSP to $\hat{X}=$ $\left\{\left(\alpha_{1} \cdot b_{i}, \ldots, \alpha_{k} \cdot b_{i}\right): b_{i} \in \mathbb{R}_{\geq 0}\right\}$ for some $\alpha \in \mathbb{R}_{>}^{n}$.

The following proposition summarizes our knowledge about the resulting simplifications, which we will refer to as $\alpha$-VCG and $\alpha$-GSP. Most of these observations were already proved or at least claimed by Milgrom.

Proposition 3. Let $\alpha \in \mathbb{R}_{>}^{n}$. Then, $\alpha$-GSP and $\alpha-V C G$ are tight on $\Theta$, have positive revenue on $\Theta^{>}$if $n, k \geq 2$, and are Vickrey-preserving on $\Theta^{\alpha}$.

Assuming that the click-through rate vector $\alpha$ is known, both $\alpha$-VCG and $\alpha$-GSP look very appealing: they eliminate all zero-revenue equilibria, without affecting the truthful equilibrium and without introducing any new equilibria.

In practice, however, the relevant click-through rates may not be known. A somewhat more realistic model assumes a certain degree of heterogeneity among the population generating the clicks. More precisely, a certain fraction of this population is assumed to be "merely curious," such that clicks by this part of the population do not generate any value for the agents. This introduces an information asymmetry, where the mechanism observes the overall clickthrough rate vector $\alpha$, while agents derive value from a different click-through rate vector $\beta{ }^{8}$ In the following we will

\footnotetext{
${ }^{8}$ As Milgrom [22] points out, "if the search company observes
} clicks but not sales or value for each position, its auction rule assume that $\beta$ is the same for all agents, and that $\beta$ is again normalized such that $\beta_{1}=1$ and $\beta_{j}=0$ if $j>k$.

For a slight variation of our model, in which there is a small dependence between $\alpha$ and $\beta,{ }^{9}$ Milgrom established a separation between GSP and VCG: $\alpha$-GSP retains the VCG outcome in situations where $\alpha$-VCG fails to do so. We obtain an analogous observation in our model. The proof of the following result is given in the full version of the paper, where we also establish that $\alpha$-VCG is usually not Vickreypreserving.

Proposition 4. Let $\alpha, \beta \in \mathbb{R}_{>}^{n}$. Then, $\alpha$-GSP is Vickrey-preserving on $\Theta^{\beta}$ if and only if the sequence $\left\{p_{j}(\theta) / \alpha_{j}\right\}_{j=1, \ldots, k}$, where $p_{j}(\theta)=\sum_{i=j}^{k}\left(v_{i+1}\left(\theta_{i+1}\right) \cdot\left(\beta_{i}-\right.\right.$ $\left.\left.\beta_{i+1}\right)\right)$, is decreasing.

But this line of reasoning seems a bit problematic for two reasons. First, there seems no reason to believe that the condition relating prices (and thus bids) and click-through rates in Proposition 4 would be satisfied in practice. Second, the above discussion only shows superiority of GSP over VCG with respect to a particular simplification, and it might well be the case that there exists a different simplification of VCG mechanism with comparable or even better properties.

An additional observation that we make, in regard to the ability of these simplifications to eliminate zero-revenue equilibria, is that there exist type profiles for which the minimum equilibrium revenue can be arbitrarily small compared to the revenue obtained in the VCG outcome.

Theorem 1. Let $\epsilon, r>0$. Then there exist $\alpha \in \mathbb{R}_{>}^{n}$ and $\theta \in \Theta^{\alpha}$ such that $R^{V C G}(\theta) \geq r$ and $\alpha-V C G$ has an equilibrium with revenue at most $\epsilon$. Similarly, there exist $\alpha \in \mathbb{R}_{>}^{n}$ and $\theta \in \Theta^{\alpha}$ such that $R^{V C G}(\theta) \geq r$ and $\alpha-G S P$ has an equilibrium with revenue at most $\epsilon$.

Proof. We consider a setting with three agents and three slots. The construction can easily be extended to an arbitrary number of agents and slots.

For $\alpha$-VCG, let $v_{i}\left(\theta_{i}\right)=r+1$ for all $i \in N$. Let $\alpha_{1}=1$, $\alpha_{2}=1 /(r+1)$, and $\alpha_{3}=1 /(2 r+2)$. It is easily verified that the bids $b_{1}=r+1$ and $b_{2}=b_{3}=\epsilon$ form a Nash equilibrium of $\alpha$-VCG. Given these bids, $\alpha$-VCG assigns slot 1 to agent 1 at price $\epsilon-\epsilon /(2 r+2)$, and slots 2 and 3 to agents 2 and 3 at prices $\epsilon /(r+1)-\epsilon /(2 r+2)$ and zero. This yields revenue $\epsilon$. In the truthful equilibrium of the VCG mechanism, on the other hand, the price is $r+\epsilon /(r+1)-\epsilon /(2 r+2)$ for the first slot, $\epsilon /(r+1)-\epsilon /(2 r+2)$ for the second slot, and zero for the third slot, for an overall revenue of $r+\epsilon /(r+2)$.

For $\alpha$-GSP, again let $v_{i}\left(\theta_{i}\right)=r+1$ for all $i \in N$. Let $\delta=\epsilon /(r+2), \alpha_{1}=1, \alpha_{2}=(1+\delta) /(r+1)$, and $\alpha_{3}=$ $1 /(r+1)$. It is easily verified that the bids $b_{1}=r+1$ and $b_{2}=b_{3}=\delta /(1+\delta) \cdot(r+1)$ form a Nash equilibrium of $\alpha$-GSP. Given these bids, $\alpha$-GSP assigns slot 1 to agent 1 at price $\delta /(1+\delta) \cdot(r+1)$, and slots 2 and 3 to agents 2 and 3 at prices $\delta$ and zero. This yields revenue $\delta /(1+\delta) \cdot(r+1)+\delta \leq \epsilon$. In the truthful equilibrium of the VCG mechanism, on the other hand, the price is $r$ for the first slot, $\delta$ for the second

can entail adjusting bids in proportion to clicks but not in proportion to value" (p. 68).

${ }^{9}$ Milgrom assumes that there is a fraction $\lambda$ of shoppers with click-through rate vector $\alpha$ and a fraction $(1-\lambda)$ of curious searchers with click-through rate vector $\beta$. The clickthrough rate vector $\gamma$ observed by the search provider is then given by $\gamma_{j}=\lambda \cdot \alpha_{j}+(1-\lambda) \cdot \beta_{j}$. 
slot, and zero for the third slot, for an overall revenue of $r+\delta$.

\subsection{A Sense in which GSP is Superior to VCG}

The above observations raise the following prominent question: does there exist a simplification that preserves the $V C G$ outcome despite ignorance about the true click-through rates that affect bidders' values, and if so, can this simplification achieve improved revenue relative to the VCG outcome, in every equilibrium?

For GSP the answer is surprisingly simple: a closer look at the proofs of Proposition 3 and Proposition 4 reveals that by ignoring the observed click-through rates $\alpha$, and setting $\alpha=$ $\mathbf{1}=(1, \ldots, 1)$ instead, one obtains a simplification that is tight, guarantees positive revenue, and is Vickrey-preserving on all of $\Psi$. This strengthens Proposition 3 over the claims for $\alpha$-GSP and $\alpha$-VCG.

COROllary 1. $\alpha$-GSP is tight on $\Psi$, has positive revenue on $\Psi$ if $n, k \geq 2$, and is Vickrey-preserving on $\Psi$, if and only if $\alpha=\mathbf{1}=(1, \ldots, 1)$.

The direction from left to right follows by observing that, for every $\alpha \neq 1$, we can find a $\beta$ such that the condition of Proposition 4 is violated.

In light of Theorem 1, and given the arguments in favor of efficient equilibria, we may further ask for the minimum revenue obtained by 1 -GSP in any efficient equilibrium. It turns out that 1-GSP always recovers at least half of the VCG revenue for all slots but the first.

TheOREM 2. Let $\beta \in \mathbb{R}_{>}^{n}, \theta \in \Theta^{\beta}$, and assume that $v_{1}\left(\theta_{1}\right) \geq$ $\cdots \geq v_{n}\left(\theta_{n}\right)$. Then, every efficient equilibrium of 1-GSP for $\theta$ yields revenue at least

$$
\frac{1}{2}\left(R^{V C G}(\theta)-\sum_{j=1}^{k}\left(\beta_{j}-\beta_{j+1}\right) \cdot v_{j+1}\left(\theta_{j+1}\right)\right) .
$$

Proof. Let $b(\theta)$ be a bid profile corresponding to an efficient equilibrium of 1-GSP. It then holds that $b_{1}(\theta) \geq \cdots \geq b_{n}(\theta)$, and for all $i \in\{1, \ldots, k\}, 1-G S P$ assigns slot $i$ to agent $i$ at price $p_{i}(\theta)=b_{i+1}(\theta)$. A necessary condition for $b(\theta)$ to be an equilibrium is that for every agent $j \in N, b_{j}(\theta)$ is large enough such that none of the agents $i>j$ would prefer being assigned slot $j$ instead of $i$. In particular, for every $i \in N$,

$$
\begin{aligned}
& \beta_{i+1} \cdot v_{i+1}\left(\theta_{i+1}\right)-p_{i+1}(\theta) \geq \beta_{i} \cdot v_{i+1}\left(\theta_{i+1}\right)-b_{i}(\theta) \quad \text { and } \\
& \beta_{i+2} \cdot v_{i+2}\left(\theta_{i+2}\right)-p_{i+2}(\theta) \geq \beta_{i} \cdot v_{i+2}\left(\theta_{i+2}\right)-b_{i}(\theta) .
\end{aligned}
$$

Since $p_{i}(\theta)=b_{i+1}(\theta)$ and by rearranging,

$$
\begin{aligned}
b_{i}(\theta) & \geq\left(\beta_{i}-\beta_{i+1}\right) \cdot v_{i+1}\left(\theta_{i+1}\right)+p_{i+1}(\theta) \\
& =\left(\beta_{i}-\beta_{i+1}\right) \cdot v_{i+1}\left(\theta_{i+1}\right)+b_{i+2}(\theta) \text { and } \\
b_{i}(\theta) & \geq\left(\beta_{i}-\beta_{i+2}\right) \cdot v_{i+2}\left(\theta_{i+2}\right)+p_{i+2}(\theta) \\
& \geq\left(\beta_{i+1}-\beta_{i+2}\right) \cdot v_{i+2}\left(\theta_{i+2}\right)+b_{i+3}(\theta) .
\end{aligned}
$$

If we repeatedly substitute according to the first inequality, we obtain

$$
\begin{aligned}
& b_{i}(\theta) \geq \sum_{j=1}^{\left\lfloor\frac{k-i}{2}\right\rfloor}\left(\beta_{i+2 \cdot j-2}-\beta_{i+2 \cdot j-1}\right) \cdot v_{i+2 \cdot j-1}\left(\theta_{i+2 \cdot j-1}\right) \quad \text { and } \\
& b_{i}(\theta) \geq \sum_{j=1}^{\left\lfloor\frac{k-i}{2}\right\rfloor}\left(\beta_{i+2 \cdot j-1}-\beta_{i+2 \cdot j}\right) \cdot v_{i+2 \cdot j}\left(\theta_{i+2 \cdot j}\right) .
\end{aligned}
$$

By adding the two inequalities,

$$
2 \cdot b_{i}(\theta) \geq \sum_{j=i}^{k}\left(\beta_{j}-\beta_{j+1}\right) \cdot v_{j+1}\left(\theta_{j+1}\right),
$$

and, since $p_{i}(\theta)=b_{i+1}(\theta)$,

$$
2 \cdot p_{i}(\theta) \geq \sum_{j=i+1}^{k}\left(\beta_{j}-\beta_{j+1}\right) \cdot v_{j+1}\left(\theta_{j+1}\right) .
$$

Now recall that $R^{V C G}(\theta)=\sum_{i \in N} r_{i}(\theta)$, where

$$
r_{i}(\theta)=\sum_{j=i}^{k}\left(\beta_{j}-\beta_{j+1}\right) \cdot v_{j+1}\left(\theta_{j+1}\right) .
$$

Thus,

$$
\sum_{i \in N} 2 \cdot p_{i}(\theta) \geq \sum_{i \in N} r_{i}(\theta)-\sum_{j=1}^{k}\left(\beta_{j}-\beta_{j+1}\right) \cdot v_{j+1}\left(\theta_{j+1}\right) .
$$

The revenue obtained in any efficient equilibrium of 1-GSP is therefore at least

$$
\sum_{i \in N} p_{i}(\theta) \geq \frac{1}{2}\left(R^{V C G}(\theta)-\sum_{j=1}^{k}\left(\beta_{j}-\beta_{j+1}\right) \cdot v_{j+1}\left(\theta_{j+1}\right)\right) .
$$

Our analysis also leads to a satisfactory contrast between 1-GSP and simplifications of VCG: any simplification of VCG that does not observe the value-generating click-through rates, and is Vickrey-preserving for all possible choices of these click-through rates, must admit an efficient equilibrium with arbitrarily low revenue.

THEOREM 3. Let $\hat{M}$ be a simplification of the VCG mechanism that is Vickrey-preserving on $\Psi$. Then, for every $\theta \in \Psi$ and every $\epsilon>0$, there exists an efficient equilibrium of $\hat{M}$ with revenue at most $\epsilon$.

Proof. Fix $\beta \in \mathbb{R}_{>}^{n}$ and consider an arbitrary type profile $\theta \in \Theta^{\beta} \subseteq \Psi$. Order the agents such that $v_{1}\left(\theta_{1}\right) \geq \cdots \geq$ $v_{n}\left(\theta_{n}\right)$.

The proof proceeds in two steps. First we will argue that for some $c \geq 0$, every $\delta>0$, and all $i \in N, \hat{X}_{i}$ must contain a message $x_{i}^{\delta}$ corresponding to bids $b_{i j}$ such that $b_{i i}=\beta_{i}$. $v_{i}\left(\theta_{i}\right)+c, b_{i j} \leq \beta_{i} \cdot v_{i}\left(\theta_{i}\right)+c+\delta$ for $1 \leq j<i$ and $b_{i j} \leq c+\delta$ for $i<j \leq k$. These messages will then be used to construct an equilibrium with low revenue.

To show that the restricted message spaces $\hat{X}_{i}$ must contain messages as described above, we show that these messages are required to reach the VCG outcome for a different type profile $\theta^{\prime} \in \Theta^{\beta^{\prime}} \subseteq \Psi$ for a particular $\beta^{\prime} \in \mathbb{R}_{>}^{n}$. Denote by $p_{i}\left(\theta^{\prime}\right)$ the price of slot $i$ for type profile $\theta^{\prime}$. We know that for $j \in\{2, \ldots, k\}, \beta_{j}^{\prime} \cdot v_{j}\left(\theta_{j}^{\prime}\right)=\beta_{j-1}^{\prime} \cdot v_{j}\left(\theta_{j}^{\prime}\right)-p_{j-1}\left(\theta^{\prime}\right)$ (see, e.g., [22]). Consider an arbitrary $\delta>0$. If we choose $\beta^{\prime}$ such that $\beta_{1}^{\prime}-\beta_{i}^{\prime}$ and $\beta_{i+1}^{\prime}$ are small enough, we can choose $\theta^{\prime}$ as above such that

$$
\begin{array}{ll}
p_{j}\left(\theta^{\prime}\right)-p_{i}\left(\theta^{\prime}\right) \leq \delta & \text { for } j<i \\
p_{i}\left(\theta^{\prime}\right)-p_{j}\left(\theta^{\prime}\right) \geq \beta_{i} \cdot v_{i}\left(\theta_{i}\right)-\delta & \text { for } j>i .
\end{array}
$$

A well-known property of the VCG outcome in the assignment problem is its envy-freeness (see, e.g., [21]): denoting 
by $b_{i j}$ the bid of agent $i$ on slot $j$ and by $p_{j}$ the price of slot $j$, it must hold for every agent $i$ that

$$
b_{i i}-p_{i} \geq b_{i j}-p_{j} \quad \text { for all } j \in S .
$$

For type profile $\theta^{\prime}$, we thus obtain

$$
\begin{array}{ll}
b_{i j}-b_{i i} \leq p_{j}\left(\theta^{\prime}\right)-p_{i}\left(\theta^{\prime}\right) \leq \delta & \text { for } j<i \text { and } \\
b_{i i}-b_{i j} \geq p_{i}\left(\theta^{\prime}\right)-p_{j}\left(\theta^{\prime}\right) \geq \beta_{i} \cdot v_{i}\left(\theta_{i}\right)-\delta & \text { for } j>i .
\end{array}
$$

Using messages $x_{i}^{\delta}$, we now construct an efficient equilibrium with low revenue. Clearly, the allocation that assigns slot $i$ to agent $i$ is still efficient under message profile $x^{\delta}$. Furthermore, for all $j \in\{1, \ldots, k\}$, the VCG price of slot $j$ under $x^{\delta}$ goes to zero as $\delta$ goes to zero. In particular, we can choose $\delta$ such that the overall revenue is at most $\epsilon$. We finally claim that there exists some $\delta^{\prime}>0$ such that $x^{\delta}$ is an equilibrium for every $\delta$ with $\delta^{\prime}>\delta>0$. To see this, recall that $\beta_{i} \cdot v_{i}\left(\theta_{i}\right)>\beta_{j} \cdot v_{i}\left(\theta_{i}\right)$ for $j>i$, so $u_{i}\left(x^{\delta}, \theta_{i}\right)>\beta_{j} \cdot v_{i}\left(\theta_{i}\right)$ for some small enough $\delta$. If, on the other hand, agent $i$ was assigned a slot $j<i$, his payment would be at least $\beta_{j} \cdot v_{j}\left(\theta_{j}\right)-\delta>\beta_{j} \cdot v_{i}\left(\theta_{i}\right)-\delta$. This would leave him with utility at most $\delta$, which can be chosen to be smaller than $u_{i}\left(x^{\delta}, \theta_{i}\right)$.

It is worth noting that despite having a reasonably good lower bound on revenue, 1-GSP does not quite succeed in circumventing Theorem 1: there exists a type profile for which only the first slot generates a significant amount of VCG revenue, and an equilibrium of 1-GSP for this type profile in which revenue is close to zero. On the other hand, for a wide range of click-through rates, there will be a large gap between the minimum revenue of 1 -GSP and simplifications of VCG in efficient equilibria.

Moreover, the revenue separation between simplifications of GSP and VCG only applies to simplifications of the latter that are Vickrey-preserving. Given how central the VCG outcome has been to the analysis of sponsored search auctions, this seems a reasonable property to impose. Nevertheless, it is an interesting question whether the result can be strengthened further.

\section{COMBINATORIAL AUCTIONS}

Mechanisms for combinatorial auctions allocate items from a set $G$ to the agents, i.e., $\Omega=\prod_{i \in N} 2^{G}$ such that for every $o \in \Omega$ and $i, j \in N$ with $i \neq j, o_{i} \cap o_{j}=\emptyset$. We make the standard assumption that the empty set is valued at zero and that valuations satisfy free disposal, i.e., for all $i \in N, \theta_{i} \in \Theta_{i}$, and $o, o^{\prime} \in \Omega, v_{i}\left(o, \theta_{i}\right)=0$ when $o_{i}=\emptyset$ and $v_{i}\left(o, \theta_{i}\right) \leq v_{i}\left(o^{\prime}, \theta_{i}\right)$ when $o_{i} \subseteq o_{i}^{\prime}$. The latter condition also implies that each agent is only interested in the package he receives, and we sometimes abuse notation and write $v_{i}\left(C, \theta_{i}\right)$ for the valuation of agent $i$ for any $o \in \Omega$ with $o_{i}=C$. We further write $k=|G|$ for the number of items, $W(o, x)=\sum_{j \in N} v_{j}\left(o, x_{j}\right)$ for the social welfare of outcome $o \in \Omega$ under message profile $x \in X$, and $W_{\max }(x)=\max _{o \in \Omega} W(o, x)$ for the maximum social welfare of any outcome. Finally, for every agent $i \in N$, message $x_{i} \in X$, and bundle of items $B \subseteq G$ we write $x_{i}(B)$ for agent $i$ 's bid on bundle $B$.

The VCG mechanism makes it a dominant strategy for every agent to bid his true valuation for every bundle of items. Since the number of such bundles is exponential in the number of items, however, computational constraints might prevent agents from playing this dominant strategy (even for a well-crafted bidding language [24, 19]). In light of these results, and in light of the observation that simplifications can help to isolate useful equilibria, it is interesting to ask which other (ex-post) equilibria the VCG auction can have. Holzman and Monderer [15] showed that these equilibria are precisely the projections of the true types to those subsets of the set of all bundles that form a quasi-field. Let $\Sigma \subseteq 2^{G}$ be a set of bundles of items such that $\emptyset \in \Sigma$. $\Sigma$ is called a quasi-field if it is closed under complementation and union of disjoint subsets, i.e., if

- $B \in \Sigma$ implies $B^{c} \in \Sigma$, where $B^{c}=G \backslash B$ and

- $B, C \in \Sigma$ and $B \cap C$ implies $B \cup C \in \Sigma$.

For a message $x_{i} \in X_{i}$, write $x_{i}^{\Sigma}$ for the projection of $x_{i}$ to $\Sigma$, i.e., for the unique message such that for every bundle $B \subseteq G$ of items,

$$
x_{i}^{\Sigma}(B)=\max _{B^{\prime} \in \Sigma, B^{\prime} \subseteq B} x_{i}\left(B^{\prime}\right) .
$$

The characterization given by Holzman and Monderer is subject to the additional constraint of variable participation: a strategy profile $s$ for a set $N$ of agents is an equilibrium of a VCG mechanism under variable participation if for every $N^{\prime} \subseteq N$, the projection of $s$ to $N^{\prime}$ is an equilibrium of every VCG mechanism for $N^{\prime}$.

Theorem 4 (Holzman and Monderer [15]). Consider a $V C G$ combinatorial auction with a set $N$ of agents and a set $G$ of items. Then, a strategy profile $s=\left(s_{1}, \ldots, s_{n}\right)$ is an ex-post equilibrium of this auction under variable participation if and only if there exists a quasi-field $\Sigma \subseteq 2^{G}$ such that for every type profile $\theta$ and every agent $i \in \bar{\epsilon} N$, $s_{i}\left(\theta_{i}\right)=\theta_{i}^{\Sigma}$.

Intuitively, the social welfare obtained in these "bundling" equilibria decreases as the set of bundles becomes smaller. A simple argument shows, for example, that welfare in the bundling equilibrium for $\Sigma$, where $|\Sigma|=2^{m}$ for some $m \leq$ $k$, can be smaller by a factor of $k / m$ than the maximum welfare. For this, consider a setting with $k$ agents such that each agent desires exactly one of the items, i.e., values this item at 1 , and each item is desired by exactly one of the agents. Clearly, maximum social welfare in this case is $k$. On the other hand, since $\Sigma$ is a quasi-field, it cannot contain more than $m$ bundles that are pairwise disjoint. Therefore, by assigning only bundles in $\Sigma$, one can obtain welfare at most $m$.

Welfare can also differ tremendously among quasi-fields of equal size, which suggests an opportunity for simplification.

Proposition 5. Let $G$ be a set of items, $k=|G|$, and $m \leq$ $k$. Then there exist quasi-fields $\Sigma, \Sigma^{\prime} \subseteq 2^{G}$ with $|\Sigma|=\left|\Sigma^{\prime}\right|=$ $2^{m}$ and a type profile $\theta$ such that

$$
\frac{W_{\max }\left(\theta^{\Sigma}\right)}{W_{\max }\left(\theta^{\Sigma^{\prime}}\right)} \geq \frac{m}{\left\lceil m^{2} / k\right\rceil} .
$$

Proof. Consider a partition of $G$ into sets $G_{1}, \ldots, G_{m}$ of size $\left\lceil\frac{k}{m}\right\rceil$ or $\left\lfloor\frac{k}{m}\right\rfloor$, and let $\Sigma$ be the closure of $\left\{G_{1}, \ldots, G_{m}\right\}$ under complementation and union of disjoint sets. For every $i, 1 \leq i \leq m$, choose an arbitrary $g_{i} \in G_{i}$, and define $\theta=\left(\theta_{1}, \ldots, \bar{\theta}_{m}\right)$ such that for every $i$ with $1 \leq i \leq m$, $v_{i}\left(X, \theta_{i}\right)=1$ if $g_{i} \in X$ and $v_{i}\left(X, \theta_{i}\right)=0$ otherwise. Clearly, $W_{\max }\left(\theta^{\Sigma}\right)=m$. Now consider a second partition of $G$ into sets $G_{1}^{\prime}, \ldots, G_{m}^{\prime}$ of size $\left\lceil\frac{k}{m}\right\rceil$ or $\left\lfloor\frac{k}{m}\right\rfloor$ such 
that $G_{j}^{\prime} \supseteq\left\{g_{i}:(j-1)\lfloor k / m\rfloor+1 \leq i \leq j\lfloor k / m\rfloor\right\}$, and let $\Sigma^{\prime}$ be the closure of $\left\{G_{1}, \ldots, G_{m}\right\}$ under complementation and union of disjoint sets. It is then easily verified that $W_{\max }\left(\theta^{\Sigma^{\prime}}\right) \leq\lceil m /(k / m)\rceil=\left\lceil m^{2} / k\right\rceil$, and the claim follows.

Moreover, agents might disagree about the quality of the different bundling equilibria of a given maximum size. In particular, the set of these equilibria might contain several Pareto undominated equilibria, but no dominant strategy equilibrium. From the point of view of equilibrium selection, this is the worst possible scenario.

Example 1. Let $N=\{1,2,3\}, G=\{A, B, C\}$, and consider a type profile $\theta=\left(\theta_{1}, \theta_{2}, \theta_{3}\right)$ such that

$$
\begin{aligned}
& v_{1}\left(X, \theta_{1}\right)= \begin{cases}4 & \text { if }\{A, C\} \subseteq X \\
3 & \text { if } A \in X \text { and } C \notin X \\
1 & \text { otherwise; }\end{cases} \\
& v_{2}\left(X, \theta_{2}\right)= \begin{cases}3 & \text { if }\{A, C\} \subseteq X \text { or }\{B, C\} \subseteq X \text { and } \\
0 & \text { otherwise; }\end{cases} \\
& v_{3}\left(X, \theta_{3}\right)= \begin{cases}1 & \text { if } B \in X \\
0 & \text { otherwise. }\end{cases}
\end{aligned}
$$

Clearly, at least four bids are required to express $\theta_{1}$. Since a quasi-field on $G$ must contain both the empty set and $G$ itself, there are four quasi-fields of size four or less:

$$
\begin{aligned}
& \Sigma^{1}=\{\emptyset,\{A\},\{B, C\},\{A, B, C\}\} \\
& \Sigma^{2}=\{\emptyset,\{B\},\{A, C\},\{A, B, C\}\} \\
& \Sigma^{3}=\{\emptyset,\{C\},\{A, B\},\{A, B, C\}\} \\
& \Sigma^{4}=\{\emptyset,\{A, B, C\}\}
\end{aligned}
$$

For $i \in\{1,2,3,4\}$, write $\theta^{i}=\left(\theta_{1}^{\Sigma^{i}}, \theta_{2}^{\Sigma^{i}}, \theta_{3}^{\Sigma^{i}}\right)$ for the projection of $\theta$ to $\Sigma^{i}$. The following is now easily verified. In the VCG outcome for $\theta^{1}$, agent 1 is assigned $\{A\}$ at price 0 for a utility of 3 , and agent 2 is assigned $\{B, C\}$ at price 1 for a utility of 2 . In the VCG outcome for $\theta^{2}$, agent 1 is assigned $\{A, C\}$ at price 3 for a utility of 1 , and agent 3 is assigned $\{B\}$ at price 0 for a utility of 1 . Finally, in the VCG outcome for $\theta^{3}$ and $\theta^{4}$, agent 1 is assigned $\{A, B, C\}$ at price 3 for a utility of 1 . The outcomes for $\theta^{1}$ and $\theta^{2}$ are both Pareto undominated. Also observe that social welfare is greater for $\theta^{1}$, while $\theta^{2}$ yields higher revenue.

Finally, the projection to a quasi-field can result in an equilibrium with revenue zero, even if revenue in the dominant strategy equilibrium is strictly positive. This is illustrated in the following example. It should be noted that this example, as well as the previous one, can easily be generalized to arbitrary numbers of agents and items and a large range of upper bounds on the size of the quasi-field.

ExAmple 2. Let $N=\{1,2,3\}, G=\{A, B, C, D\}$, and consider a type profile $\theta=\left(\theta_{1}, \theta_{2}, \theta_{3}\right)$ such that

$$
\begin{aligned}
& v_{1}\left(X, \theta_{1}\right)= \begin{cases}2 & \text { if }\{A, D\} \subseteq X \text { and } \\
0 & \text { otherwise }\end{cases} \\
& v_{2}\left(X, \theta_{2}\right)= \begin{cases}2 & \text { if }\{A, B\} \subseteq X \\
1 & \text { if } B \in X \text { and } A \notin X, \text { and } \\
0 & \text { otherwise; }\end{cases}
\end{aligned}
$$

$$
v_{3}\left(X, \theta_{3}\right)= \begin{cases}2 & \text { if } C \in X, \text { and } \\ 0 & \text { otherwise. }\end{cases}
$$

In the VCG outcome for $\theta$, agent 1 is assigned $\{A, D\}$ at price 1 , agent 2 is assigned $\{B\}$ at price 0 , and agent 3 is assigned $\{C\}$ at price 0 , for an overall revenue of 1 . In the VCG outcome for $\theta^{\Sigma}$, on the other hand, where $\Sigma=$ $\{\emptyset, A B, C D, A B C D\}$, agent 2 is assigned $\{A, B\}$ and agent 3 is assigned $\{C, D\}$, both at price 0 . Revenue is 0 as well.

In theory, one way to solve these problems is to simplify the mechanism, and artificially restrict the set of bundles agents can bid on. Given a set $\Sigma \subseteq 2^{G}$ of bundles, call $\Sigma$-VCG the simplification of the VCG mechanism obtained by restricting the message spaces to $\hat{X}_{i} \subseteq X_{i}$ such that for every $\hat{x}_{i} \in \hat{X}_{i}$ and every bundle of items $B \subseteq G$,

$$
\hat{x}_{i}(B)=\max _{B^{\prime} \in \Sigma, B^{\prime} \subseteq B} \hat{x}_{i}\left(B^{\prime}\right) .
$$

In other words, $\Sigma$-VCG allows agents to bid only on elements of $\Sigma$ and derives bids for the other bundles as the maximum bid on a contained bundle. It is easy to see that $\Sigma$-VCG is maximal in range [23], i.e., that it maximizes social welfare over a subset of $\Omega$. It follows that for each agent, truthful projection onto $\Sigma$ is a dominant strategy in $\Sigma$-VCG.

This shows that simplification can focus attention on a focal (truthful) equilibrium and thus avoid equilibrium selection along a Pareto frontier. As Proposition 5 and the above examples suggest, this can have a significant positive impact on both social welfare and revenue. It does not tell us, of course, how $\Sigma$ should be chosen in practice. One understood approach for maximizing social welfare without any knowledge about the quality of different outcomes, and without consideration to $\Sigma$ being a quasi-field, is to partition $G$ arbitrarily into $m$ sets of roughly equal size, where $m$ is the largest number of bundles agents can bid on. The welfare thus obtained is smaller than the maximum social welfare by a factor of at most $k / \sqrt{m}$ [16]. If additional knowledge is available, however, it may be possible to improve the result substantially, as the above results comparing the outcomes for different values of $\Sigma$ show.

With that being said, there are (at least) two additional properties that are desirable for a simplification: that $\Sigma$ VCG is tight, and that $\Sigma$ is a quasi-field. Tightness ensures that no additional equilibria are introduced as compared to the fully expressive VCG mechanism, such that the quality of the worst equilibrium outcome of the simplification is no worse than that of the original mechanism. This remains important when $\Sigma$ may still be too large for agents to use its full projection, in which case agents would again have to select from a large set of possible ex-post equilibria. By requiring that $\Sigma$ is a quasi-field, in addition to being a dominant strategy equilibrium of $\Sigma$-VCG, truthful projection to $\Sigma$ is also an ex-post equilibrium of the fully expressive VCG mechanism, and thus stable against unrestricted unilateral deviations. This ensures that agents do not experience regret, in the sense of being prevented from sending a message they would want to send given the messages sent by the other agents.

It turns out that these two requirements are actually equivalent, i.e., that $\Sigma$-VCG is tight if and only if $\Sigma$ is a quasi-field. The following result holds with respect to both Nash equilibria and ex-post equilibria. 
TheOrem 5. Let $\Sigma \subseteq 2^{G}$ such that $\emptyset \in \Sigma$. Then, $\Sigma-V C G$ is a tight simplification if $\Sigma$ is a quasi-field, and this condition is also necessary if $n \geq 3$.

Proof. For the direction from right to left, assume that $\Sigma$ is a quasi-field. By Proposition 1 it suffices to show that $\Sigma$-VCG satisfies outcome closure. Fix valuation functions $v_{j}$ and types $\theta_{j}$ for all $j \in N$, and consider an arbitrary agent $i \in N$. We claim that for every $x_{i} \in X_{i}$ and every $\hat{x}_{-i} \in \hat{X}_{-i}$,

$$
u_{i}\left(\left(\theta_{i}^{\Sigma}, \hat{x}_{-i}\right), \theta_{i}\right) \geq u_{i}\left(\left(x_{i}, \hat{x}_{-i}\right), \theta_{i}\right) .
$$

To see this, observe that there exists $\hat{\theta}_{-i} \in \Theta_{-i}$ such that $\hat{\theta}_{-i}^{\Sigma}=\hat{x}_{-i}$, and consider the type profile $\left(\theta_{i}, \hat{\theta}_{-i}\right)$. Holzman et al. [16] have shown that the projection of the true types to a quasi-field $\Sigma$ is an ex-post equilibrium of the (fully expressive) VCG mechanism. Thus, in particular, $\theta_{i}^{\Sigma}$ is a best response to $\hat{\theta}_{-i}^{\Sigma}=\hat{x}_{-i}$, which proves the claim.

For the direction from left to right, assume that $\Sigma$ is not a quasi-field. Holzman et al. [16] have shown that in this case, $\theta^{\Sigma}$ is not an ex-post equilibrium of the VCG mechanism. On the other hand, $\theta^{\Sigma}$ is a dominant-strategy equilibrium of $\Sigma$-VCG, because $\Sigma$-VCG is maximal in range. This shows that $\Sigma$-VCG is not tight.

Together with Theorem 4, this yields a characterization of the ex-post equilibria of $\Sigma$-VCG for the case when $\Sigma$ is a quasi-field.

Corollary 2. Let $\Sigma$ be a quasi-field. Then, $\hat{x}$ is an expost equilibrium of $\Sigma-V C G$ under variable participation if and only if $\hat{x}=\theta^{\Sigma^{\prime}}$ for some quasi-field $\Sigma^{\prime} \subseteq \Sigma$.

Since a quasi-field of size $m$ can contain at most $\log m$ bundles that are pairwise disjoint, insisting that a simplification be tight does come at a cost, decreasing the worst-case social welfare in the truthful projection by an additional factor of up to $\sqrt{m / \log m}$. Still, as discussed above, tightness brings other advantages to the simplified mechanism.

\section{THE ROLE OF INFORMATION}

Like most of the literature on sponsored search auctions, we have assumed that agents have complete information about each others' valuations for the different slots. It turns out that this assumption is crucial, and that the positive results for $\alpha$-GSP do not extend to incomplete information settings.

In particular, $\alpha$-GSP admits an ex-post equilibrium only in very degenerate cases. This complements a result of Gomes and Sweeney [13], who showed that $\alpha$-GSP has an efficient Bayes-Nash equilibrium on type space $\Theta^{\alpha}$ if and only if $\alpha$ decreases sufficiently quickly. Of course, ex-post equilibrium is stronger than Bayes-Nash equilibrium, but on the other hand our result precludes the existence of the former for a significantly larger type space. The proofs of all results in this section are given in the full version of the paper.

Theorem 6. Let $\alpha, \beta \in \mathbb{R}_{>}^{n}$. Then, $\alpha-G S P$ has an efficient ex-post equilibrium on type space $\Theta^{\beta}$ if and only if $n \leq 2$ or $k \leq 1$, even if $\alpha=\beta$.

The $\alpha$-VCG mechanism does only slightly better: it has an ex-post equilibrium only when it allows agents to bid truthfully (it is thus not a meaningful simplification, in that the language is in this sense exact).
THEOREM 7. Let $\alpha, \beta \in \mathbb{R}_{>}^{n}$. Then, $\alpha-V C G$ has an efficient ex-post equilibrium on type space $\Theta^{\beta}$ if and only if $\alpha=\beta$, or $n \leq 2$, or $k \leq 1$.

These results indicate that simplification is not very useful in sponsored search auctions without the assumption of complete information amongst bidders. Interestingly, simplification is also not necessary in this case to preclude equilibria with bad properties, at least in the special case of our model where the valuations are proportional to some (possibly unknown) vector of value-generating click-through rates. In particular, payments in every efficient ex-post equilibrium of the fully expressive VCG mechanism equal the VCG payments. Moreover, truthful reporting is the only efficient expost equilibrium when the number of agents is greater than the number of slots.

Proposition 6. Consider a VCG sponsored search auction with type profile $\theta \in \Theta^{\alpha}$, and assume that $s=\left(s_{1}, \ldots, s_{n}\right)$ is an efficient ex-post equilibrium. Then, for all $i$ with $1 \leq$ $i \leq k$, the payment for slot $i$ in the outcome for strategy profile $s$ equals $p_{i}=\sum_{j=i}^{\min (k, n-1)}\left(\left(\alpha_{j}-\alpha_{j+1}\right) \cdot v_{j+1}\left(\theta_{j+1}\right)\right)$. Moreover, if $n>k$, then $s_{i}\left(k, \theta_{i}\right)=\alpha_{k} \cdot v_{i}\left(\theta_{i}\right)$ for all $i$ with $1 \leq i \leq n$.

In considering simplifications for combinatorial auctions, we have adopted the standard approach to assume incomplete information amongst agents, and in particular discussed a family of simplifications of the VCG mechanism that offers a tradeoff between social welfare and the amount of information agents have to communicate.

One may wonder why this tradeoff is necessary, and in how far it depends on the amount of information agents have about each others' types. It turns out that in the complete information case a much smaller number of bids is enough to preserve an efficient equilibrium, and in fact all equilibria, of the fully expressive VCG mechanism.

We show this using a simplification of the VCG mechanisms that we call $n$-VCG. The message space of $n$-VCG consists of all bid vectors with at most $n$ non-zero entries, where $n$ is the number of agents. This reduces the number of bids elicited from each agent from $2^{k}$ to at most $n$, which can be exponentially smaller. Surprisingly, this simplification is both tight and total, i.e., the set of Nash equilibria is completely unaffected by this restriction of the message space.

THEOREM 8. The $n$-VCG mechanism is tight and total with respect to Nash equilibria.

\section{CONCLUSION AND FUTURE WORK}

In this paper we have studied simplifications of mechanisms obtained by restricting their message space, and have found that they can be used to solve different kinds of equilibrium selection problems that occur in practice. Direct revelation mechanisms typically have several equilibria, which might be more or less desirable from the point of view of the designer. Computational constraints might also imply that only a subset of the equilibria of a mechanisms can actually be achieved, in which case agents might have to select among several Pareto optimal equilibria. On the other hand, restricting the message space of a mechanism often reduces the amount of social welfare that can be achieved theoretically, and this seems to pivot on whether or not agents have complete information about each others' types. The choice 
between mechanisms with different degrees of expressiveness therefore involves a tradeoff between a benefit of simplicity and a price of simplicity.

The price of simplicity can easily be quantified, for example, by the loss in social welfare potentially incurred by a simplification, and has been studied in the context of both sponsored search and combinatorial auctions. Abrams et al. [1] and Blumrosen et al. [7], among others, have given bounds on the loss of social welfare incurred by $\alpha$ GSP for different classes of valuations that are not proportional to the click-through rates. Christodoulou et al. [8] and Bhawalkar and Roughgarden [5] have studied the potential loss in welfare when a set of items is sold through a simplification of the combinatorial auction in which only bids that are additive in items are allowed. This bidding language requires only a small number of bids, and welfare in equilibrium turns out to be smaller by at most a logarithmic factor in the number of agents than the optimum achieved by the fully expressive mechanism.

The benefit of simplicity is much harder to grasp as a concept. In this paper we have argued that simplification can improve the economic properties of a mechanism by precluding bad or promoting good equilibria. A remaining challenge is to understand the benefit of simplicity in the context of simplified mechanisms for which the computation of an equilibrium might be an intractable problem, like the ones of Christodoulou et al. and Bhawalkar and Roughgarden described above. In contrast to the simplifications considered in the present paper, these mechanisms may not be able to solve the computational or informational problem of enabling agents to bid in a straightforward way. More generally, it is far from obvious how "straightforwardness" of a mechanism should be measured, but it seems reasonable to require that agents' strategies can be computed in polynomial time.

\section{REFERENCES}

[1] Z. Abrams, A. Ghosh, and E. Vee. Cost of conciseness in sponsored search auctions. In Proc. of 3rd WINE, volume 4858 of $L N C S$, pages 326-334, 2007.

[2] L. M. Ausubel and P. Milgrom. The lovely but lonely Vickrey auction. In P. Cramton, Y. Shoham, and P. Steinberg, editors, Combinatorial Auctions, chapter 1, pages 17-40. MIT Press, 2006.

[3] M. Babaioff and T. Roughgarden. Equilibrium efficiency and price complexity in sponsored search auctions. In Proc. of 6th Workshop on Ad Auctions, 2010.

[4] M. Benisch, N. Sadeh, and T. Sandholm. A theory of expressiveness in mechanisms. In Proc. of 23rd AAAI Conference, pages 17-23, 2008.

[5] K. Bhawalkar and T. Roughgarden. Welfare guarantees for combinatorial auctions with item bidding. In Proc. of 22nd SODA, pages 700-709, 2011.

[6] L. Blumrosen, N. Nisan, and I. Segal. Auctions with severely bounded communication. Journal of Artificial Intelligence Research, 28:233-266, 2007.

[7] L. Blumrosen, J. Hartline, and S. Nong. Position auctions and non-uniform conversion rates. In Proc. of 4 th Workshop on Ad Auctions, 2008.

[8] G. Christodoulou, A. Kovács, and M. Schapira. Bayesian combinatorial auctions. In Proc. of 35th ICALP, pages 820-832, 2008.
[9] E. H. Clarke. Multipart pricing of public goods. Public Choice, 11:17-33, 1971.

[10] V. Conitzer and T. Sandholm. Computational criticisms of the revelation principle. In Proc. of 4 th $A C M$ EC Conference, pages 262-263, 2004.

[11] B. Edelman, M. Ostrovsky, and M. Schwartz. Internet advertising and the generalized second price auction: Selling billions of dollars worth of keywords. American Economic Review, 97(1):242-259, 2007.

[12] M. Feldman and L. Blumrosen. Implementation with a bounded action space. In Proc. of 7th ACM-EC Conference, pages 62-71, 2006.

[13] R. D. Gomes and K. S. Sweeney. Bayes-Nash equilibria of the generalized second price auction. In Proc. of 10 th ACM-EC Conference, pages 107-108, 2009.

[14] T. Groves. Incentives in teams. Econometrica, 41:617631, 1973.

[15] R. Holzman and D. Monderer. Characterization of ex post equilibrium in the VCG combinatorial auctions. Games and Economic Behavior, 47:87-103, 2004.

[16] R. Holzman, N. Kfir-Dahav, D. Monderer, and M. Tennenholtz. Bundling equilibrium in combinatorial auctions. Games and Economic Behavior, 47:104-123, 2004.

[17] N. Hyafil and C. Boutilier. Partial revelation automated mechanism design. In Proc. of 22nd AAAI Conference, pages 72-78, 2007.

[18] N. Hyafil and C. Boutilier. Mechanism design with partial revelation. In Proc. of 20th IJCAI, pages 13331340, 2007.

[19] S. Lahaie and D. C. Parkes. On the communication requirements of verifying the VCG outcome. In Proc. of 9th ACM-EC Conference, pages 108-113, 2008.

[20] S. Lahaie, D. M. Pennock, A. Saberi, and R. V. Vohra. Sponsored search auctions. In N. Nisan, T. Roughgarden, E. Tardos, and V. Vazirani, editors, Algorithmic Game Theory, chapter 28, pages 699-716. Cambridge University Press, 2007.

[21] H. B. Leonard. Elicitation of honest preferences for the assignment of individuals to positions. Journal of Political Economy, 91(3):461-479, 1983.

[22] P. Milgrom. Simplified mechanisms with an application to sponsored-search auctions. Games and Economic Behavior, 70(1):62-70, 2010.

[23] N. Nisan and A. Ronen. Computationally feasible VCG mechanisms. Journal of Artificial Intelligence Research, 29:19-47, 2007.

[24] N. Nisan and I. Segal. The communication requirements of efficient allocations and supporting prices. Journal of Economic Theory, 129(1):192-224, 2006.

[25] S. Shakkottai, R. Srikant, A. Ozdaglar, and D. Acemoglu. The price of simplicity. IEEE Journal on Selected Areas in Communications, 26(7):1269-1276, 2008.

[26] H. Varian. Position auctions. International Journal of Industrial Organization, 25:1163-1178, 2007.

[27] W. Vickrey. Counterspeculation, auctions, and competitive sealed tenders. Journal of Finance, 16(1):8-37, 1961. 